\title{
Non-Linear Relationship of Gamma-Glutamyl Transpeptidase To Lymphocyte Count Ratio With The Recurrence of Hepatocellular Carcinoma with Staging I-II: A Retrospective Cohort Study
}

\section{Zeping Li}

Zhengzhou University First Affiliated Hospital

Lili Liang

Zhengzhou University First Affiliated Hospital

\section{Wen Duan}

Zhengzhou University First Affiliated Hospital

\section{Chengmao Zhou}

Zhengzhou University First Affiliated Hospital

Jianjun Yang ( $\square$ zepingli1025@163.com )

Zhengzhou University First Affiliated Hospital https://orcid.org/0000-0002-0686-7719

\section{Research Article}

Keywords: hepatocellular carcinoma, gamma-glutamyl transpeptidase to lymphocyte count ratio, recurrence, no-linear.

Posted Date: August 9th, 2021

DOl: https://doi.org/10.21203/rs.3.rs-770193/v1

License: (9) (1) This work is licensed under a Creative Commons Attribution 4.0 International License.

Read Full License

Version of Record: A version of this preprint was published at Infectious Agents and Cancer on April 8th, 2022. See the published version at https://doi.org/10.1186/s13027-022-00428-0. 


\section{Abstract \\ Background}

high recurrence rate was a major factor for poor postoperative prognosis of hepatocellular carcinoma (HCC) patients. The present study was intended to evaluate the association of gamma-glutamyl transpeptidase to lymphocyte count ratio (GLR) and the recurrence of HCC with staging I-II in Chinese.

\section{Methods}

the retrospective cohort data was derived from the First Affiliated Hospital of Zhengzhou University from January 2014 to December 2018 on 496 patients who underwent radical resection of HCC with staging III. Multivariable Cox regression models were used to determine hazard ratios (HR) and $95 \%$ confidence intervals (Cls) for the recurrence of HCC with staging I-II of each GLR tertile category. The restricted cubic spline model was used to find out the threshold effect.

\section{Results}

with the low tertile of GLR as the reference, multivariable-adjusted HRs and $95 \%$ Cls of the middle and high tertile categories were $1.748(1.170,2.612)$ and $2.078(1.339,3.227)$. In addition, there was a positive correlation $(1.002(1.001,1.004))$ and a non-liner relationship was found, whose point was 27.5 . When the GLR was less than 27.5 , the risk of recurrence increased, obviously with the increase in GLR levels (1.041 $(1.014,1.068))$.

\section{Conclusions}

the GLR was independently associated with the recurrence of HCC patients with staging I-II. Furthermore, the relationship was positive and no-linear.

\section{Introduction}

Hepatocellular carcinoma (HCC) accounted for $75 \%-85 \%$ of primary liver cancer and was one of the leading causes of death related to tumor in the world[1]. In China, it accounted for $46.7 \%$ of global cases of liver cancer in 2018[2]. Compared with HCC patients with staging III-IV (American Joint Committee on Cancer pathological TNM stage, 7th ), there were more choices for treatments for HCC patients with staging I-II. Surgical resection may be the first therapeutic option for HCC patients in stages I-II, who have a better prognosis after surgery than HCC patients in stages III-IV [3, 4]. Despite advancements in HCC early detection, surgical technology, and postoperative monitoring, the overall prognosis of HCC patients after radical resection has remained unsatisfactory due to the high postoperative recurrence rate [5]. 
Therefore, it was vital to adequately understand the risk factors for the recurrence of HCC patients with staging I-II, which can be used to guide clinical decision making and improve patient prognosis.

As we all know, inflammatory and immune factors both played important roles in carcinogenesis and tumor recurrence[6, 7]. Meanwhile, blood routine and liver function were essential in blood biochemical tests for HCC patients in hospital, so needed inflammatory markers were readily available. Recently, some researchers have proposed that the aspartate aminotransferase to neutrophil ratio index (ANRI), aspartate aminotransferase to platelet ratio index (APRI), platelet to albumin ratio (PAR) and neutrophil times $\mathrm{Y}^{-}$ glutamyl transpeptidase to lymphocyte ratio (NyLR) were independent effective predictors of the prognosis of patients with HCC [8-11]. Similarly, the preoperative gamma-glutamyl transferase to lymphocyte ratio (GLR) has been found to have significant prognostic value after resection in HCC patients with single tumor size $\leq 5 \mathrm{~cm}$ and with AFP-negative[12-14]. However, the prognostic value of GLR has not been reported in the monitoring of the recurrence of HCC patients with staging I-II following curative resection.

Therefore, the purpose of the retrospective cohort study was to evaluate the association between GLR and the recurrence of HCC patients with staging $\mathrm{III}$ and to explore their dose-response relationship, which may provide guidance for the development of postoperative therapeutic approaches.

\section{Materials And Methods \\ 2.1 Study Population}

From January, 2014 to December, 2018, 496 HCC patients who underwent radical resection were enrolled in the study at the First Affiliated Hospital of Zhengzhou University. The retrospective study was approved by the Medical Ethics Committee of the First Affiliated Hospital of Zhengzhou University. The data was anonymous, and the need for informed consent was therefore waived. The patients who met the following criteria were included in this study: (1) The postoperative pathological result was hepatocellular

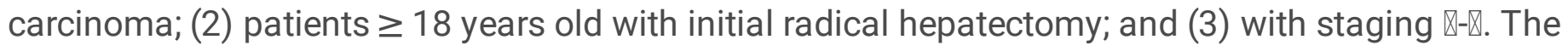
patients who met the following criteria were ruled out: (1) HCC complicated by other fatal diseases or primary tumors; (2) Patients who received preoperative chemotherapy and/or radiotherapy; (3) Patients who had a disease of the immune system or blood system.

\subsection{Follow up and data extraction}

The follow-up time ended in July, 2019, lost to follow-up or death. Follow-up was conducted every three months after surgery. The postoperative patients who did not come to our hospital for reexamination were followed up by telephone. The baseline information included age, sex, smoking, drinking, Child-Pugh class, HCC family history, operation time, differentiation, microvascular invasion, ASA (American Society of Aneshesiologists physical status classification system), urine volume, blooding volume, allogeneic blood, Hemoglobin (HB), platelet (PLT), lymphocyte count, gamma-glutamyl transpeptidase (GGT), alanine aminotransferase (ALT), aspartate aminotransferase (AST), alkaline phosphatase (ALP), hepatitis 
B surface antigen (HBsAg), tumor number; tumor-node-metastasis (TNM), tumor size, alpha-fetoprotein (AFP), and recurrence. Recurrence was defined as the presence of clinical symptoms correlated with hepatic ultrasound, computed tomography (CT) or serum alpha-fetoprotein abnormal conditions.

\subsection{Statistical Analysis}

The primary aim of the analysis was to explore the association between GLR and the recurrence of HCC

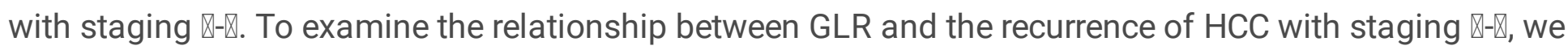
performed three different models using the univariate and multivariate Cox proportional-hazards regression model, including the non-adjusted model (no covariates were adjusted), adjusted model $₫$ (adjusted for age and sex) and adjusted model $\square$ (covariates were included as potential confounder in model $\mathbb{Q}$ if they changed the estimates of GLR on recurrence by more than $10 \%$ or were significantly associated with recurrence). Effect sizes with 95\% confidence intervals were recorded. For baseline characteristics analysis, the chi-square tested for categorical variables, the one-way ANOVA tested for continuous variables, or the Kruskal-Whallis tested for skewed distribution were used for the evaluation of the differences among the tertiles of GLR. Data was expressed as mean \pm standard deviation (SD) (Gaussian distribution) or median (min, max) (Skewed distribution) for continuous variables, and frequency or percentage for categorical variables.

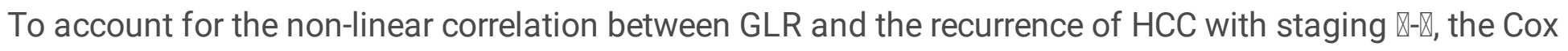
proportional hazards regression model with cubic spline functions and the smooth curve fitting (penalized spline method) were used to address nonlinearity. In addition, a two-piece-wise Cox proportional-hazards regression model was used to find the threshold effect.

We performed stratified analyses to explore potential indicators that may modify the relationship between GLR and the recurrence of HCC with staging $\mathbb{X}-\mathbb{X}$. The modification across subgroups was inspected by the likelihood ration test. Finally, we converted GLR into a categorical variable according to the tertiles of GLR, and calculated the $P$ for trend in order to confirm the results of GLR as a continuous variable, and to examine the nonlinearity.

Data analyses used R (http://www.R-project.org) and Empower(R) (www.empowerstats.com, X\&Y solutions, Inc., Boston, MA). P values $<0.05$ (two-sided) were considered statistically significant.

\section{Results}

\subsection{Subject Characteristics}

Characteristics of the study patients stratified by GLR tertiles were displayed in Table 1. Of $496 \mathrm{HCC}$ patients included in the final analyses, $199(40.121 \%)$ patients developed recurrences. A total of 165 patients were in the low GLR group (tertile 1: GLR <20.442), 165 patients were in the middle GLR group (tertile 2: $20.442-50.382$ ), and 166 patients were in the high-GLR group (tertile $3: G L R \geq 50.382$ ). The subjects included 102 women and 394 men. Patients with high GLR values (GLR $\geq 50.382$ ) were more 
likely to receive allogeneic blood therapy and to report a history of smoking and drinking; they also had

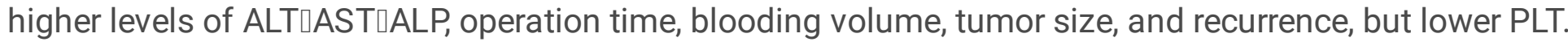


Table 1

Baseline characteristics of the study patients according to the tertiles of GLR(n $=496)$

\begin{tabular}{|c|c|c|c|c|}
\hline Variables & $\begin{array}{l}\text { Tertile } 1 \\
(<20.442, n=165)\end{array}$ & $\begin{array}{l}\text { Tertile } 2 \\
(20.442-50.382, \mathrm{n}= \\
165)\end{array}$ & $\begin{array}{l}\text { Tertile } 3 \\
(>50.382, n=166)\end{array}$ & $\begin{array}{l}\mathrm{P}- \\
\text { value }\end{array}$ \\
\hline Age (years) & & & & 0.133 \\
\hline$\leq 60$ & $111(67.273 \%)$ & $113(68.485 \%)$ & $127(76.506 \%)$ & \\
\hline$>60$ & $54(32.727 \%)$ & 52 (31.515\%) & $39(23.494 \%)$ & \\
\hline Sex & & & & 0.003 \\
\hline Male & 117 (70.909\%) & $135(81.818 \%)$ & $142(85.542 \%)$ & \\
\hline Female & $48(29.091 \%)$ & 30 (18.182\%) & $24(14.458 \%)$ & \\
\hline Smoking & & & & $\begin{array}{l}<.001 \\
0.001\end{array}$ \\
\hline No & $123(74.545 \%)$ & $102(61.818 \%)$ & $91(54.819 \%)$ & \\
\hline Yes & $42(25.455 \%)$ & $63(38.182 \%)$ & $75(45.181 \%)$ & \\
\hline Drinking & & & & 0.015 \\
\hline NO & $140(84.848 \%)$ & $128(77.576 \%)$ & $119(71.687 \%)$ & \\
\hline Yes & $25(15.152 \%)$ & 37 (22.424\%) & $47(28.313 \%)$ & \\
\hline HCC family history & & & & 0.666 \\
\hline No & $142(86.061 \%)$ & 147 (89.091\%) & $147(88.554 \%)$ & \\
\hline Yes & $23(13.939 \%)$ & 18 (10.909\%) & $19(11.446 \%)$ & \\
\hline HBsAg & & & & 0.744 \\
\hline Negative & $24(14.545 \%)$ & $21(12.727 \%)$ & $26(15.663 \%)$ & \\
\hline Positive & $139(84.242 \%)$ & $139(84.242 \%)$ & $137(82.530 \%)$ & \\
\hline NA & $2(1.212 \%)$ & $5(3.030 \%)$ & $3(1.807 \%)$ & \\
\hline $\operatorname{ALT}(\mathrm{U} / \mathrm{L})$ & $\begin{array}{l}23.000(8.000- \\
101.000)\end{array}$ & $\begin{array}{l}28.000(10.000- \\
265.000)\end{array}$ & $\begin{array}{l}38.000(10.000- \\
605.000)\end{array}$ & $\begin{array}{l}< \\
0.001\end{array}$ \\
\hline AST (U/L) & $\begin{array}{l}24.000(12.000- \\
89.000)\end{array}$ & $\begin{array}{l}29.000(14.000- \\
159.000)\end{array}$ & $\begin{array}{l}40.500(15.000- \\
386.000)\end{array}$ & ¿. 001 \\
\hline
\end{tabular}

Notes: data presented were mean $\pm S D$, median $(\mathrm{Q} 1-\mathrm{Q} 3)$, or $\mathrm{N}(\%)$. Abbreviations: ALT, alanine aminotransferase; AST, aspartate aminotransferase; ALP, alkaline phosphatase; Hb, Hemoglobin; PLT, platelet; AFP, alpha-fetoprotein; HBsAg, hepatitis B surface antigen; ASA, American society of Aneshesiologists physical status classification system; TNM, tumor-node-metastasis; GLR, gammaglutamyl transpeptidase to lymphocyte count ratio. 


\begin{tabular}{|c|c|c|c|c|}
\hline Variables & $\begin{array}{l}\text { Tertile } 1 \\
(<20.442, \mathrm{n}=165)\end{array}$ & $\begin{array}{l}\text { Tertile } 2 \\
(20.442-50.382, n= \\
165)\end{array}$ & $\begin{array}{l}\text { Tertile } 3 \\
(>50.382, n=166)\end{array}$ & $\begin{array}{l}\mathrm{P} \text { - } \\
\text { value }\end{array}$ \\
\hline $\operatorname{ALP}(\mathrm{U} / \mathrm{L})$ & $77.058(22.856)$ & 87.962 (29.539) & $116.245(68.271)$ & $\dot{c}_{0.001}$ \\
\hline $\mathrm{HB}(\mathrm{g} / \mathrm{L})$ & $134.727(15.461)$ & $135.273(17.457)$ & $131.333(20.427)$ & 0.096 \\
\hline PLT (10^9/L) & $153.073(51.750)$ & $136.170(57.049)$ & 124.476 (69.775) & $\begin{array}{l}< \\
0.001\end{array}$ \\
\hline $\operatorname{AFP}(\mathrm{ng} / \mathrm{ml})$ & & & & 0.071 \\
\hline$\leq 20$ & $70(44.872 \%)$ & $82(51.572 \%)$ & $62(38.750 \%)$ & \\
\hline$>20$ & $86(55.128 \%)$ & 77 (48.428\%) & $98(61.250 \%)$ & \\
\hline Child-Pugh class & & & & 0.065 \\
\hline A & $162(98.182 \%)$ & $156(94.545 \%)$ & $154(92.771 \%)$ & \\
\hline B & $3(1.818 \%)$ & 9 (5.455\%) & $12(7.229 \%)$ & \\
\hline Differentiation & & & & 0.615 \\
\hline Well-moderate & $144(91.139 \%)$ & $145(90.062 \%)$ & $137(87.821 \%)$ & \\
\hline Poor & $14(8.861 \%)$ & $16(9.938 \%)$ & 19 (12.179\%) & \\
\hline $\begin{array}{l}\text { Lymphovascular } \\
\text { invasion }\end{array}$ & & & & 0.224 \\
\hline No & $130(78.788 \%)$ & 137 (83.030\%) & $125(75.301 \%)$ & \\
\hline Yes & $35(21.212 \%)$ & $28(16.970 \%)$ & 41 (24.699\%) & \\
\hline ASA & & & & 0.377 \\
\hline ० & $19(11.585 \%)$ & $13(7.927 \%)$ & $19(11.446 \%)$ & \\
\hline ० & 137 (83.537\%) & $135(82.317 \%)$ & $136(81.928 \%)$ & \\
\hline ० & $8(4.878 \%)$ & $16(9.756 \%)$ & $11(6.627 \%)$ & \\
\hline $\begin{array}{l}\text { Operation time } \\
\text { (min) }\end{array}$ & $140.745(46.511)$ & $166.097(58.854)$ & $177.175(63.115)$ & $\dot{0.001}$ \\
\hline Urine volume(ml) & $\begin{array}{l}300.00(0.000- \\
1200.000)\end{array}$ & $\begin{array}{l}350.000(20.000- \\
3500.000)\end{array}$ & $\begin{array}{l}300.000(50.000- \\
2150.000)\end{array}$ & 0.069 \\
\hline
\end{tabular}

Notes: data presented were mean \pm SD, median (Q1-Q3), or $N(\%)$. Abbreviations: ALT, alanine aminotransferase; AST, aspartate aminotransferase; ALP, alkaline phosphatase; Hb, Hemoglobin; PLT, platelet; AFP, alpha-fetoprotein; HBsAg, hepatitis B surface antigen; ASA, American society of Aneshesiologists physical status classification system; TNM, tumor-node-metastasis; GLR, gammaglutamyl transpeptidase to lymphocyte count ratio. 


\begin{tabular}{|c|c|c|c|c|}
\hline Variables & $\begin{array}{l}\text { Tertile } 1 \\
(<20.442, n=165)\end{array}$ & $\begin{array}{l}\text { Tertile } 2 \\
(20.442-50.382, n= \\
165)\end{array}$ & $\begin{array}{l}\text { Tertile } 3 \\
(>50.382, n=166)\end{array}$ & $\begin{array}{l}\mathrm{P}- \\
\text { value }\end{array}$ \\
\hline $\begin{array}{l}\text { Bleeding } \\
\text { volume(ml) }\end{array}$ & $\begin{array}{l}200.000(5.000- \\
1400.000)\end{array}$ & $\begin{array}{l}300.000(20.000- \\
3500.000)\end{array}$ & $\begin{array}{l}400.000(20.000- \\
5000.000)\end{array}$ & $\dot{0} 001$ \\
\hline Allogeneic blood & & & & $\begin{array}{l}<.001 \\
0.001\end{array}$ \\
\hline No & $156(95.122 \%)$ & $146(89.571 \%)$ & $122(73.494 \%)$ & \\
\hline Yes & $8(4.878 \%)$ & $17(10.429 \%)$ & $44(26.506 \%)$ & \\
\hline Tumor number & & & & 0.864 \\
\hline Single & 154 (93.333\%) & $154(93.333 \%)$ & $157(94.578 \%)$ & \\
\hline Multiple & $11(6.667 \%)$ & $11(6.667 \%)$ & $9(5.422 \%)$ & \\
\hline TNM & & & & 0.183 \\
\hline I & $122(73.939 \%)$ & $128(77.576 \%)$ & $114(68.675 \%)$ & \\
\hline II & $43(26.061 \%)$ & $37(22.424 \%)$ & $52(31.325 \%)$ & \\
\hline Tumor size $(\mathrm{cm})$ & & & & $\hat{0}_{0.001}$ \\
\hline$\leq 5$ & $132(80.000 \%)$ & $108(65.455 \%)$ & $92(55.422 \%)$ & \\
\hline$>5$ & $33(20.000 \%)$ & $57(34.545 \%)$ & $74(44.578 \%)$ & \\
\hline Recurrence & & & & $\begin{array}{l}<.001 \\
0.00\end{array}$ \\
\hline No & $120(72.727 \%)$ & $93(56.364 \%)$ & $84(50.602 \%)$ & \\
\hline Yes & $45(27.273 \%)$ & $72(43.636 \%)$ & $82(49.398 \%)$ & \\
\hline \multicolumn{5}{|c|}{$\begin{array}{l}\text { Notes: data presented were mean } \pm \text { SD, median }(\mathrm{Q} 1-\mathrm{Q} 3) \text {, or } \mathrm{N}(\%) \text {. Abbreviations: ALT, alanine } \\
\text { aminotransferase; AST, aspartate aminotransferase; ALP, alkaline phosphatase; Hb, Hemoglobin; PLT, } \\
\text { platelet; AFP, alpha-fetoprotein; HBsAg, hepatitis B surface antigen; ASA, American society of } \\
\text { Aneshesiologists physical status classification system; TNM, tumor-node-metastasis; GLR, gamma- } \\
\text { glutamyl transpeptidase to lymphocyte count ratio. }\end{array}$} \\
\hline
\end{tabular}

\subsection{Association of non-recurrence and recurrence of HCC with staging I-II}

In the univariate analysis, sex, smoking, HBsAg, ALT, AST, ALP, GLR, AFP, vascular invasion, blooding volume, allogeneic blood, TNM and tumor size were significantly associated with the recurrence of HCC with staging I-II (Table 2). 
Page $9 / 20$ 
Table 2

Univariate analysis to identify risk factors associated with the recurrence of HCC patients with staging I- II

\begin{tabular}{|c|c|c|c|}
\hline Variables & statistics & $\mathrm{HR} 95 \% \mathrm{Cl}$ & P Value \\
\hline \multicolumn{4}{|l|}{ Age (years) } \\
\hline$\leq 60$ & $351(70.766 \%)$ & 1.0 & \\
\hline$>60$ & 145 (29.234\%) & $0.742(0.540,1.020)$ & 0.06634 \\
\hline \multicolumn{4}{|l|}{ Sex } \\
\hline Male & 394 (79.435\%) & 1.0 & \\
\hline Female & $102(20.565 \%)$ & $0.638(0.435,0.937)$ & 0.02180 \\
\hline \multicolumn{4}{|l|}{ Smoking } \\
\hline No & 316 (63.710\%) & 1.0 & \\
\hline Yes & 180 (36.290\%) & $1.346(1.016,1.783)$ & 0.03872 \\
\hline \multicolumn{4}{|l|}{ Drinking } \\
\hline No & 387 (78.024\%) & 1.0 & \\
\hline Yes & 109 (21.976\%) & $1.208(0.878,1.662)$ & 0.24598 \\
\hline \multicolumn{4}{|c|}{ HCC family history } \\
\hline No & 436 (87.903\%) & 1.0 & \\
\hline Yes & 60 (12.097\%) & $1.248(0.837,1.861)$ & 0.27741 \\
\hline \multicolumn{4}{|l|}{$\mathrm{HBsAg}$} \\
\hline Negative & 71 (14.609\%) & 1.0 & \\
\hline Positive & 415 (85.391\%) & $1.627(1.024,2.583)$ & 0.03917 \\
\hline $\operatorname{ALT}(\mathrm{U} / \mathrm{L})$ & $38.823 \pm 42.262$ & $1.002(0.999,1.004)$ & 0.20455 \\
\hline $\mathrm{AST}(\mathrm{U} / \mathrm{L})$ & $37.423 \pm 30.174$ & $1.005(1.001,1.008)$ & 0.00610 \\
\hline $\operatorname{ALP}(U / L)$ & $93.767 \pm 47.817$ & $1.006(1.004,1.009)$ & $<0.00001$ \\
\hline GLR & $55.969 \pm 74.589$ & $1.003(1.002,1.004)$ & $<0.00001$ \\
\hline $\mathrm{HB}(\mathrm{g} / \mathrm{L})$ & $133.773 \pm 17.953$ & $1.011(1.003,1.019)$ & 0.01016 \\
\hline PLT (10^9/L) & $137.879 \pm 61.046$ & $1.001(0.998,1.003)$ & 0.62340 \\
\hline \multicolumn{4}{|l|}{ AFP (ng/ml) } \\
\hline$\leq 20$ & $214(45.053 \%)$ & 1.0 & \\
\hline
\end{tabular}




\begin{tabular}{|c|c|c|c|}
\hline Variables & statistics & $\mathrm{HR}$ 95\%Cl & P Value \\
\hline$>20$ & $261(54.947 \%)$ & $1.605(1.199,2.150)$ & 0.00149 \\
\hline \multicolumn{4}{|l|}{ Child-Pugh class } \\
\hline A & 472 (95.161\%) & 1.0 & \\
\hline B & 24 (4.839\%) & $1.050(0.572,1.930)$ & 0.87432 \\
\hline \multicolumn{4}{|l|}{ Differentiation } \\
\hline Well-moderate & 426 (89.684\%) & 1.0 & \\
\hline Poor & 49 (10.316\%) & $0.932(0.586,1.484)$ & 0.76792 \\
\hline \multicolumn{4}{|c|}{ Lymphovascular invasion } \\
\hline No & 392 (79.032\%) & 1.0 & \\
\hline Yes & $104(20.968 \%)$ & $2.337(1.705,3.204)$ & $<0.00001$ \\
\hline \multicolumn{4}{|l|}{ ASA } \\
\hline ૫ & 51 (10.324\%) & 1.0 & \\
\hline प & $408(82.591 \%)$ & $1.007(0.638,1.587)$ & 0.97718 \\
\hline प & $35(7.085 \%)$ & $0.947(0.482,1.864)$ & 0.87588 \\
\hline Operation time (min) & $161.371 \pm 58.525$ & $1.002(1.000,1.005)$ & 0.03542 \\
\hline Urine volume(ml) & $371.830 \pm 256.635$ & $1.000(1.000,1.001)$ & 0.39094 \\
\hline Blooding volume (ml) & $397.597 \pm 479.510$ & $1.000(1.000,1.000)$ & 0.18443 \\
\hline \multicolumn{4}{|l|}{ Allogeneic blood } \\
\hline No & $424(86.004 \%)$ & 1.0 & \\
\hline Yes & $69(13.996 \%)$ & $1.542(1.070,2.223)$ & 0.02024 \\
\hline \multicolumn{4}{|l|}{ Tumor number } \\
\hline Single & $465(93.750 \%)$ & 1.0 & \\
\hline Mutiple & $31(6.250 \%)$ & $1.885(1.127,3.150)$ & 0.01565 \\
\hline \multicolumn{4}{|l|}{ TNM } \\
\hline$\square$ & $364(73.387 \%)$ & 1.0 & \\
\hline प & $132(26.613 \%)$ & $2.443(1.813,3.292)$ & $<0.00001$ \\
\hline \multicolumn{4}{|l|}{ Tumor size $(\mathrm{cm})$} \\
\hline$\leq 5$ & 332 (66.935\%) & 1.0 & \\
\hline
\end{tabular}




\begin{tabular}{|llll|}
\hline Variables & statistics & HR 95\%Cl & P Value \\
\hline$>5$ & $164(33.065 \%)$ & $1.633(1.229,2.170)$ & 0.00071 \\
\hline
\end{tabular}

\subsection{Association of GLR and the recurrence of HCC with staging I-II}

Table 3 showed that the association between GLR and the risk of recurrence was determined. With adjustment for potential confounders, increased GLR had a positive relationship with the recurrence of HCC with staging I-II $(1.002(1.001,1.004))$. In the adjusted model II, the hazard ratios $(95 \% \mathrm{Cl})$ for the recurrence of HCC with staging I-II across GLR tertiles were as follows: The low tertile: $<20.442(1.0)$, the middle tertile: $20.442-50.382(1.748(1.170,2.612))$, the high tertile: $>50.382(2.078(1.339,3.227))$, independent of age, sex, smoking, operation time, lymphovascular invasion, allogeneic blood, HB, AST, ALP, HBsAg, tumor number, tumor size, AFP.

Table 3

Association between GLR and the risk of recurrence of HCC with staging I-II

\begin{tabular}{|c|c|c|c|}
\hline Exposure & Non-adjusted & Adjust I & Adjust II \\
\hline GLR & $\begin{array}{l}1.003(1.002,1.004)< \\
0.00001\end{array}$ & $\begin{array}{l}1.003(1.002,1.004)< \\
0.00001\end{array}$ & $\begin{array}{l}1.002(1.001,1.004) \\
0.00884\end{array}$ \\
\hline \multicolumn{4}{|l|}{$\begin{array}{l}\text { GLR } \\
\text { tertiles }\end{array}$} \\
\hline Low & 1.0 & 1.0 & 1.0 \\
\hline Middle & $\begin{array}{l}1.899(1.308,2.758) \\
0.00075\end{array}$ & $\begin{array}{l}1.827(1.254,2.660) \\
0.00168\end{array}$ & $\begin{array}{l}1.748(1.170,2.612) \\
0.00644\end{array}$ \\
\hline High & $\begin{array}{l}2.375(1.646,3.426)< \\
0.00001\end{array}$ & $\begin{array}{l}2.263(1.565,3.273) \\
0.00001\end{array}$ & $\begin{array}{l}2.078(1.339,3.227) \\
0.00112\end{array}$ \\
\hline $\begin{array}{l}P \text { for } \\
\text { trend }\end{array}$ & $<0.00001$ & 0.00001 & 0.00127 \\
\hline \multicolumn{4}{|c|}{$\begin{array}{l}\text { Notes: data presented were HRs and } 95 \% \text { Cls. Non-adjusted model adjusted for: None; Adjust I model } \\
\text { adjusted for: age; sex; Adjust II model adjusted for: age; sex; smoking; operation time; lymphovascular } \\
\text { invasion: allogeneic blood: HB: AST: ALP: HBsAq: Tumor number: Tumor size; AFP. }\end{array}$} \\
\hline
\end{tabular}

\subsection{Threshold Effect Analysis of GLR on incident recurrence of HCC with staging I-II}

To confirm whether a dose-response relation between GLR and incident recurrence of HCC with staging I-II existed, we performed a smoothing function analysis. After adjusting for age, sex, smoking, operation 
time, lymphovascular invasion, allogeneic blood, HB, AST, ALP, HBsAg, tumor number, tumor size and AFP, a nonlinear relationship between GLR and incident recurrence was found (Fig. 1). Using a two-piecewise Cox proportional-hazards regression model, we found that incident recurrence was positively correlated with the GLR until it peaked at 27.5 [HR $1.041(1.014,1.068), P=0.0028]$. However, when the GLR was higher than 27.5, the hazard ratio for risk of developing recurrence was $1.001(1.000,1.003)$, indicating that there was no significant association between the risk of developing recurrence and increased GLR ( $P$ $=0.0997)($ Table 4$)$.

In Fig. 1, the full line indicated the estimated risk of incident recurrence, and the vacant lines represented a point-wise $95 \%$ confidence interval, adjusted for age, sex, smoking, HCC family history, operation time, lymphovascular invasion, allogeneic blood, HB, AST, ALP, HBsAg, tumor number, tumor size, AFP.

Table 4

Threshold effect analysis of GLR on incident recurrence of HCC with staging I-II

\begin{tabular}{|llc|}
\hline Outcome: Recurrence & HR (95\%IC) & P value \\
\hline One-line linear regression model & $1.002(1.001,1.004)$ & 0.0088 \\
\hline Two-piecewise linear regression model & & \\
\hline GLR $\leq 27.5$ & $1.041(1.014,1.068)$ & 0.0028 \\
\hline GL > 27.5 & $1.001(1.000,1.003)$ & 0.0997 \\
\hline Log-likelihood ratio test & 0.003 & \\
\hline $\begin{array}{l}\text { Notes: adjusted for age; sex; smoking; operation time; lymphovascular invasion; allogeneic blood; HB; } \\
\text { AST; ALP; HBsAg; tumor number; tumor size; AFP. }\end{array}$ & \\
\hline
\end{tabular}

\subsection{Subgroup Analyses}

HBsAg $(P=0.0341)$ and AFP $(P=0.0416)$ were interaction factors between GLR and the recurrence of $\mathrm{HCC}$ with staging I-II. Limiting the analysis to HBsAg negative patients showed a significant positive relationship between GLR and recurrence (HR 1.007(1.003, 1.012), $P=0.0023)$, whereas this relationship was no longer significant in HBsAg positive patients. Similarly, the positive relationship was significant in AFP-negative $(\leq 20 \mathrm{ng} / \mathrm{ml})$ patients, but was not significant in AFP-positive ( $>20 \mathrm{ng} / \mathrm{ml})$ patients. After adjustment for potential confounding variables, we found that the relationship between GLR and recurrence did not change by age, sex, allogeneic blood, lymphovascular invasion, tumor number, tumor size (all $P$ for interaction $>0.05$ ) (Table 5). 
Table 5

Subgroup analysis of the association between GLR and the risk of recurrence of HCC with staging I-II

$\begin{array}{lllllll}\text { Parameters } & \text { N } & \text { HR } & 95 \% \mathrm{Cl} \text { Low } & 95 \% \mathrm{Cl} \text { High } & \text { P value } & \mathrm{P} \text { (interaction) }\end{array}$

Age (years)

$\begin{array}{lllll} & & & & \\ 329 & 1.002 & 1.000 & 1.004 & 0.0313 \\ 133 & 1.003 & 1.000 & 1.007 & 0.0664\end{array}$

$\leq 60$

0.4760

$>60$

Sex

Male

$\begin{array}{lll}367 & 1.002 & 1.000\end{array}$

1.004

0.0169

Female

$\begin{array}{lll}95 & 1.003 & 0.998\end{array}$

1.008

0.2005

Smoking

No

$\begin{array}{lll}298 & 1.001 & 0.999\end{array}$

1.004

0.3412

Yes

$\begin{array}{lll}164 & 1.003 & 1.001\end{array}$

1.005

0.0047

Allogeneic blood

No

$\begin{array}{lll}396 & 1.002 & 1.000\end{array}$

1.003

0.1524

Yes

$\begin{array}{lll}66 & 1.005 & 1.001\end{array}$

1.010

0.0514

HBsAg

Negative

$69 \quad 1.007 \quad 1.003$

1.012

0.0341

Positive

$\begin{array}{lll}393 & 1.002 & 1.000\end{array}$

1.003

0.0209

$\operatorname{AFP}(\mathrm{ng} / \mathrm{ml})$

$\leq 20$

$208 \quad 1.005 \quad 1.002$

1.009

0.0014

0.0416

$>20$

$\begin{array}{lll}254 & 1.002 & 1.000\end{array}$

1.003

0.1016

Lymphovascular invasion

No

$\begin{array}{lll}362 & 1.001 & 0.999\end{array}$

1.003

0.2805

Yes

$100 \quad 1.004 \quad 1.001$

1.006

0.0011

Tumor number

Single

Multiple

Tumor size $(\mathrm{cm})$

$\begin{array}{lll}431 & 1.002 & 1.000\end{array}$

1.003

0.0662

$\begin{array}{lll}31 & 1.006 & 1.002\end{array}$

1.009

0.0011

0.0980

0.2988 


\begin{tabular}{|lccllll|}
\hline Parameters & N & HR & 95\%Cl Low & 95\%Cl High & P value & P (interaction) \\
\hline$\leq 5$ & 309 & 1.003 & 1.001 & 1.005 & 0.0057 \\
\hline$>5$ & 153 & 1.001 & 0.999 & 1.004 & 0.2617 \\
\hline $\begin{array}{l}\text { Notes: adjust for age; sex; smoking; operation time; lymphovascular invasion; allogeneic blood; HB; } \\
\text { AST; ALP; HBsAg; tumor number; TNM; tumor size; AFP. }\end{array}$ \\
\hline
\end{tabular}

\section{Discussion}

Our study found that elevated GLR has a positive correlation with the recurrence of HCC patients with staging $\mathbb{\square} \square$ after adjustment of age, sex, smoking, operation time, lymphovascular invasion, allogeneic blood, HB, AST, ALP, HBsAg, tumor number, tumor size, AFP. To the best of our knowledge, we were the first to discover a nonlinear relationship between GLR and the risk of recurrence in HCC patients at stage $\mathrm{I}-\mathrm{II}$, with a cutoff point of 27.5 . The nonlinear relationship was as follows: the hazard ratio $(95 \% \mathrm{Cl})$ of incident recurrence was $1.041(1.014,1.068)$ when the GLR was less than 27.5 and $1.001(1.000,1.003)$ when the GLR was beyond 27.5. Moreover, according to the subgroup analysis, the association between

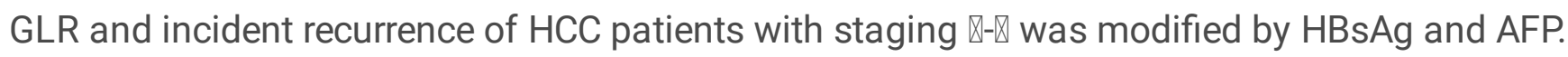

Inflammatory responses played a vital role in all stages of cancer development and progression [15-17]. In the early stages of cancers, inflammatory cell recruitment created a favorable microenvironment for tumor growth and facilitated the formation of new blood vessel formation[18, 19]. Intrahepatic GGT was chiefly found on the surface of the cell and played a key role in glutathione metabolism[20]. Furthermore, previous studies have shown that increased preoperative increased GGT values were positive relationship with a poor prognosis of hepatocellular carcinoma [21-23]. GGT had pro-oxidant effect and catalyzed the generation of reactive oxygen species (ROS), ROS played proinflammatory function in the the NF-KB signaling pathway $[22,24,25]$. Similarly, lymphocytes inhibited tumor proliferation and migration in the human immune system, and increased lymphocyte count predicted favorable prognosis in patients with various solid tumors [26-28].

In recent years, the prediction model of prognosis of the HCC combined with different inflammation factors has always been a hot issue[9, 10,29,30]. Previous studies have examined GLR's prognostic ability in HCC [14, 31], intrahepatic cholangiocarcinoma [32] and nonfunctional pancreatic neuroendocrine tumors[25]. These studies revealed that increased GLR values had a positive correlation with poor longterm outcomes, which matched our findings. However, building a reliable model required us to understand the true relationship between each predictor and the prognosis of $\mathrm{HCC}$, where a non-linear relationship was important. In this study, we found that the relationship between GLR and the recurrence of HCC with staging $\mathbb{\otimes} \mathbb{\square}$ was nonlinear. This would help us build models in the future.

Subsequently, by subgroup analysis, the association between GLR and the recurrence of HCC with staging $\mathbb{X} \mathbb{X}$ was modified by HBsAg and AFP. In HBsAg-negative patients, our study found a significant positive relationship, but this relationship was closely significant $(P=0.0589)$ in $\mathrm{HBsAg}$ - positive patients. 
The previous study found that in HCC patients with AFP-negative after radical resection, GLR had fair accuracy in predicting the early-recurrence, and our findings persisted [13].

Noticeably, to our knowledge, the nonlinear relationship between GLR and the recurrence in HCC with staging $\mathbb{\nabla} \mathbb{\square}$ was first found. This study, however, has some limitations. First, this study was retrospective and included patients from a single-center hospital. Second, only patients who underwent first resection and with staging $\mathbb{\otimes}-\mathbb{W}$ were included in this study. Furthermore, the results of the study were for the Chinese population and may not be applicable to people in other countries. Due to these limitations, multicenter and large-scale studies were warranted to validate our finds.

In conclusion, the increased GLR was independently associated with the recurrence of HCC patients with staging I-II. Furthermore, the relationship between increased GLR and developing recurrence was positive, no-linear and modified by HBsAg and AFP.

\section{Declarations}

\section{Ethical approval and consent to participate}

The study was approved by the Ethics Committee of the Medical Ethics Committee of the First Affiliated Hospital of Zhengzhou University. The data was anonymous, and the need for informed consent was therefore waived.

\section{Consent for publication}

Not applicable.

\section{Availability of data and materials}

The data that support the findings of this study are available on request from the corresponding author: Jianjun Yang, Department of Anesthesiology, Pain and Perioperative Medicine, The First Affiliated Hospital of Zhengzhou University, Zhengzhou, 450000, China.

\section{Competing interests}

The authors declare that they have no competing interests.

\section{Funding}

Not applicable.

\section{Authors' contributions}

All authors contributed to the study design, the primary analysis, and manuscript preparation.

\section{Acknowledgements}


Not applicable.

\section{References}

1. Bray FFJ, Soerjomataram ISR, Torre LAJA. Global Cancer S. 2018: GLOBOCAN Estimates of Incidence and Mortality Worldwide for 36 Cancers in 185 Countries. CA Cancer J Clin. 2018;68(6):394-424.

2. Feng R, Zong Y, Cao S, Xu R. Current cancer situation in China: good or bad news from the 2018 Global Cancer Statistics? Cancer Commun. 2019;39(1):22.

3. Liu P, Hsu C, Hsia C, Lee Y, Su C, Huang Y, et al. Prognosis of hepatocellular carcinoma: Assessment of eleven staging systems. J HEPATOL. 2016;64(3):601-8.

4. Shi M, Jiang Y, Yang L, Yan S, Wang YG, Lu XJ. Decreased levels of serum exosomal miR-638 predict poor prognosis in hepatocellular carcinoma. J CELL BIOCHEM. 2018;119(6):4711-6.

5. Wen T, Jin C, Facciorusso A, Donadon M, Han H, Mao Y, et al. Multidisciplinary management of recurrent and metastatic hepatocellular carcinoma after resection: an international expert consensus. HEPATOBIL SURG NUTR. 2018 2018-01-01;7(5):353-71.

6. Lu C, Rong D, Zhang B, Zheng W, Wang X, Chen Z, et al. Current perspectives on the immunosuppressive tumor microenvironment in hepatocellular carcinoma: challenges and opportunities. MOL CANCER. 2019;18(1).

7. Sanghera C, Teh JJ, Pinato DJ. The systemic inflammatory response as a source of biomarkers and therapeutic targets in hepatocellular carcinoma. LIVER INT. 2019;39(11):2008-23.

8. Ji F, Fu S, Guo Z, Pang H, Chen D, Wang X, et al. Prognostic significance of preoperative aspartate aminotransferase to neutrophil ratio index in patients with hepatocellular carcinoma after hepatic resection. Oncotarget. 2016 2016-01-01;7(44):72276-89.

9. Allenson K, Roife D, Kao LS, Ko TC, Wray CJ. Estimation of hepatocellular carcinoma mortality using aspartate aminotransferase to platelet ratio index. Journal of gastrointestinal oncology. 20202020 01-01;11(2):291-7.

10. Li C, Peng W, Zhang X, Wen T, Chen L. The preoperative platelet to albumin ratio predicts the prognosis of hepatocellular carcinoma patients without portal hypertension after liver resection. MEDICINE. 2019;98(45):e17920.

11. Li J, Liao Y, Suo L, Zhu P, Chen X, Dang W, et al. A novel prognostic index-neutrophil times Yglutamyl transpeptidase to lymphocyte ratio (NyLR) predicts outcome for patients with hepatocellular carcinoma. SCI REP-UK. 2017;7(1).

12. Liao M, Qin W, Liao Y, Yao R, Yu J, Liao W. Prognostic Value of Gamma-Glutamyl Transpeptidase to Lymphocyte Count Ratio in Patients With Single Tumor Size $\leq 5 \mathrm{~cm}$ Hepatocellular Carcinoma After Radical Resection. FRONT ONCOL. 2019 2019-05-21;9.

13. Li S, Xu W, Liao M, Zhou Y, Weng J, Ren L, et al. The Significance of Gamma-Glutamyl Transpeptidase to Lymphocyte Count Ratio in the Early Postoperative Recurrence Monitoring and Prognosis Prediction of AFP-Negative Hepatocellular Carcinoma. 2021;Volume 8:23-33. 
14. Zhang H, Zhou Y, Li Y, Qin W, Zi Y, Liu Y, et al. Predictive value of gamma-glutamyl transpeptidase to lymphocyte count ratio in hepatocellular carcinoma patients with microvascular invasion. BMC CANCER. 2020;20(1).

15. Qian B. Inflammation fires up cancer metastasis. SEMIN CANCER BIOL. 2017 2017-01-01;47:170-6.

16. Singh R, Mishra MK, Aggarwal H. Inflammation, Immunity, and Cancer. MEDIAT INFLAMM. 2017;2017:1.

17. Greten FR, Grivennikov SI. Inflammation and Cancer: Triggers, Mechanisms, and Consequences. IMMUNITY. 2019 2019-01-01;51(1):27-41.

18. Coffelt SB, de Visser KE. Inflammation lights the way to metastasis. NATURE. [Journal Article; Comment]. 2014 2014-03-06;507(7490):48-9.

19. Sarvaiya PJGD, Ulasov IGPLM. Chemokines in tumor progression and metastasis. Oncotarget. 2013;4(12):2171-85.

20. Ikeda YTN. Gene expression of gamma-glutamyltranspeptidase. Methods Enzymol. 2005;401:40825.

21. Zhang $L, L v Y, X u A$, Wang $H$. The prognostic significance of serum gamma-glutamyltransferase levels and AST/ALT in primary hepatic carcinoma. BMC CANCER. 2019;19(1).

22. Xia J, Song P, Sun Z, Sawakami T, Jia M, Wang Z. Advances of diagnostic and mechanistic studies of $\mathrm{Y}$-glutamyl transpeptidase in hepatocellular carcinoma. Drug Discoveries Therapeutics. 2016;10(4):181-7.

23. Wang Z, Song P, Xia J, Inagaki Y, Tang W, Kokudo N. Can gamma-glutamyl transferase levels contribute to a better prognosis for patients with hepatocellular carcinoma? Drug Discoveries Therapeutics. 2014;8(3):134-8.

24. Stark AA, Russell JJ, Langenbach R, Pagano DA, Zeiger E, Huberman E. Localization of oxidative damage by a glutathione-gamma-glutamyl transpeptidase system in preneoplastic lesions in sections of livers from carcinogen-treated rats. CARCINOGENESIS. [Journal Article; Research Support Non-US. Gov't; Research Support, U.S. Gov't, Non-P.H.S.; Research Support, U.S. Gov't, P.H.S.]. 1994 1994-02-01;15(2):343-8.

25. Zhou B, Zhan C, Wu J, Liu J, Zhou J, Zheng S. Prognostic significance of preoperative gammaglutamyltransferase to lymphocyte ratio index in nonfunctional pancreatic neuroendocrine tumors after curative resection. SCI REP-UK. 2017;7(1).

26. Zhang J, Huang S, Li H, Li Y, Chen X, Zhang W, et al. Preoperative lymphocyte count is a favorable prognostic factor of disease-free survival in non-small-cell lung cancer. MED ONCOL. 2013;30(1).

27. Milne K, Alexander C, Webb JR, Sun W, Dillon K, Kalloger SE, et al. Absolute lymphocyte count is associated with survival in ovarian cancer independent of tumor-infiltrating lymphocytes. J TRANSL MED. 2012;10(1):33. 2012-01-01.

28. Feng F, Zheng G, Wang Q, Liu S, Liu Z, Xu G, et al. Low lymphocyte count and high monocyte count predicts poor prognosis of gastric cancer. BMC GASTROENTEROL. 2018;18(1). 
29. Guo L, Ren H, Pu L, Zhu X, Liu Y, Ma X. The Prognostic Value of Inflammation Factors in Hepatocellular Carcinoma Patients with Hepatic Artery Interventional Treatments: A Retrospective Study. CANCER MANAG RES. 2020 2020-01-01;12:7173-88.

30. Liu K, Lv Y, Niu Y, Bu Y. Prognostic value of Y-glutamyl transpeptidase to albumin ratio combined with aspartate aminotransferase to lymphocyte ratio in patients with hepatocellular carcinoma after hepatectomy. MEDICINE. 2020 2020-11-25;99(48):e23339.

31. Liao M, Qin W, Liao Y, Yao R, Yu J, Liao W. Prognostic Value of Gamma-Glutamyl Transpeptidase to Lymphocyte Count Ratio in Patients With Single Tumor Size $\leq 5 \mathrm{~cm}$ Hepatocellular Carcinoma After Radical Resection. FRONT ONCOL. 2019 2019-05-21;9.

32. Wang JJLH, Li JXXLWH. Preoperative gamma-glutamyltransferase to lymphocyte ratio predicts longterm outcomes in intrahepatic cholangiocarcinoma patients following hepatic resection. World $\mathrm{J}$ Gastroenterol. 2020;26(13):1501-12.

\section{Figures}




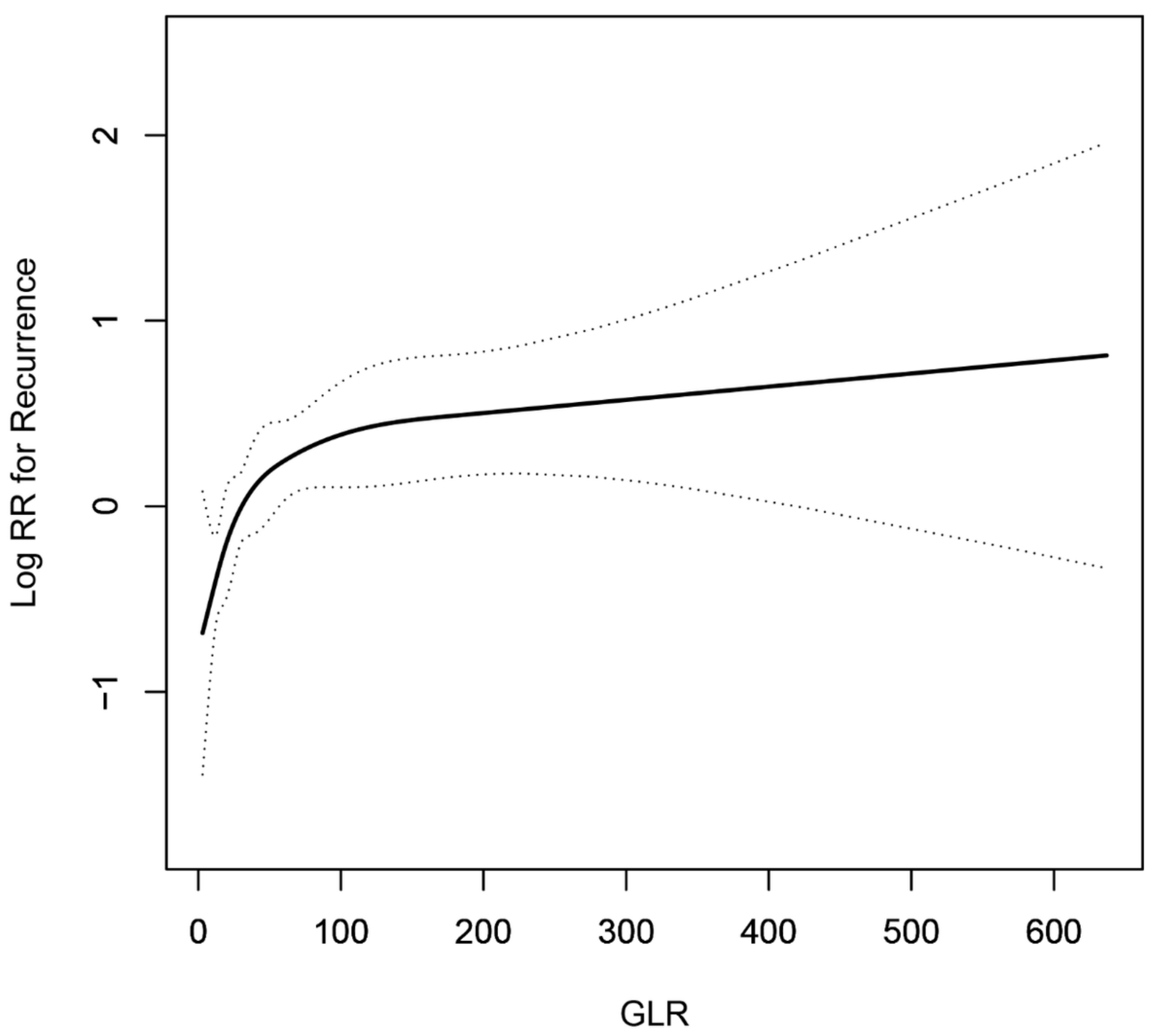

Figure 1

Dose-response relationship between GLR and the risk of recurrence of HCC patients with staging I-II 